\title{
Computics Approach toward Clarification of Atomic Reactions during Epitaxial Growth of GaN
}

\author{
Atsushi Oshiyama ${ }^{1}$, Kieu My Bui ${ }^{1}$, Mauro Boero ${ }^{2,1}$, Yoshihiro Kangawa ${ }^{3,1}$, and Kenji Shiraishi ${ }^{1,4}$ \\ ${ }^{1}$ Institute of Materials and Systems for Sustainability, Nagoya University, Nagoya 464-8601, Japan \\ \{oshiyama, bui.my\}@imass.nagoya-u.ac.jp \\ ${ }^{2}$ University of Strasbourg, Institut de Physique et Chimie des Matériaux de Strasbourg, CNRS, UMR 7504, F67034, Strasbourg, France \\ mauro.boero@ipcms.unistra.fr \\ ${ }^{3}$ Research Institute for Applied Mechanics, Kyushu University, Fukuoka 816-8580, Japan \\ kangawa@riam.kyushu-u.ac.jp \\ ${ }^{4}$ Graduate School of Engineering, Nagoya University, Nagoya 464-8603, Japan \\ shiraishi@cse.nagoya-u.ac.jp
}

\begin{abstract}
We report first-principles calculations based on the density-functional theory that clarify atomic reactions of ammonia decomposition and subsequent nitrogen incorporation during GaN epitaxial growth. We find that Ga-Ga weak bonds are ubiquitous on Ga-rich growing surface and responsible for the growth reactions. Furthermore, Car-Parrinello Molecular Dynamics simulations predict the existence of 2-dimensional Ga liquid phase, providing new insight into the epitaxial growth. The obtained results are expected to become basics for multiscale growth simulations in future.
\end{abstract}

Keywords-density functional theory, Car-Parrinello molecular dynamics, GaN, epitaxial growth

\section{INTRODUCTION}

Density-functional theory (DFT) [1] combined with the Kohn-Sham (KS) scheme [2] was innovated more than a halfcentury ago and then provided a new way to study physical and chemical properties of real materials quantum mechanically. Subsequent progress in computational methods allows us to apply this KSDFT scheme to a numerous number of materials and unprecedented success has been achieved [3]. In addition to reveal atomic and electronic properties of stable and metastable structures, dynamical properties have been also explored by introducing molecular dynamics (MD) scheme. In particular, the MD combined with DFT proposed by Car and Parrinello (CPMD) is proved to be particularly powerful [4-6].

Performance of supercomputers increases exponentially and now it reaches 410 PFLOPS (Fugaku in Japan) as its theoretical peak performance [7]. This situation of the computers seems to reinforce the computational approach. However, it is not necessarily true. The performance of a single compute node is already saturated. Hence the only way to make a high-performance computer is to gather a huge number of compute nodes and connect them with high-speed network (massively parallel architecture). Moreover hardware accelerators have been introduced to perform a part of the scientific calculations. Hence, without the cross-disciplinary collaboration among the field of physical science or device simulation and the field of computer science or applied mathematics, it would be impossible to exploit the tremendous performance of the supercomputers. We call this new stage of the collaboration computics [8].

In this article, we present our efforts to clarify microscopic mechanisms of Metal-Organic Vapor-Phase Epitaxy (MOVPE) of gallium nitride (GaN) [9] based on the DFT calculations. These efforts just get started and preliminary in computics viewpoints but the obtained results are already interesting [10-12]. We find that the existence of Ga-Ga weak bonds on the growing surface is essential for the epitaxial growth.

GaN known as a premier material in optoelectronics is now emerging in power electronics due to its wider band-gap, higher mobility and robustness under harsh environment [13]. In GaN MOVPE, trimethylgallium (TMG) and ammonia $\left(\mathrm{NH}_{3}\right)$ are used as gas sources. Many experimental and theoretical efforts have revealed that TMG is decomposed in the gas phase and the growing surface is generally Ga rich [1416]. On the other hand, the mechanism of the decomposition of $\mathrm{NH}_{3}$ is unclear though it is postulated without any evidence to be decomposed in the gas phase and forms several $\mathrm{NH}_{\mathrm{x}}$ unit [17]. On the contrary, recent high-resolution time-of-flight (TOF) measurements [16] show that ammonia is rarely decomposed in the gas phase: Only small amount $(0.1 \%)$ of $\mathrm{NH}_{2}$ is detected in the gas phase except for $\mathrm{NH}_{3}$. Hence clarification of the atomistic behavior of $\mathrm{NH}_{3}$ on the growing $\mathrm{GaN}$ surface is the first priority to be attacked by the DFT calculations.

\section{COMPUTATIONAL METHODS}

All the calculations have been performed in DFT with the generalized gradient approximation [18] to the many-body exchange-correlation energy functional. Nuclei and core electrons are described by norm-conserving pseudo-potentials [19]. The Ga 3d states are treated as core-electron states with or without the partial core correction [20]. The geometryoptimized electronic-structure computations have been done using our real-space-scheme code called RSDFT [21] which has been developed so as to achieve the state-of-the-art highperformance computing [22]. Dynamical simulations have been performed by Car-Parrinello molecular dynamics (CPMS) as is implemented in the plane-wave basis-set scheme [23]. The GaN (0001) surface is simulated by a repeating slab model in which each slab consisting of six GaN double layers are separated from its images by the $15-\AA$ thick vacuum regions. The bottom $\mathrm{N}$ surface is passivated by fractionally charged $(0.75 e)$ hydrogen to annihilate the dangling bonds and mimic the semi-infinite $\mathrm{GaN}$ substrate [24]. Other calculational details and their validity are found in References 10 and 12. Reaction pathways and corresponding energy barriers are calculated by using the hyperplane constraint method (HPC) [25]. 


\section{DECOMPOSITION OF $\mathrm{NH}_{3}$ AND INCORPORATION OF $\mathrm{N}$ ON GAN(0001)}

Thermodynamical analyses combined with DFT calculations [15] have shown that the growing surface in GaN MOVPE at the typical range of temperature and pressure of gas sources is Ga rich. We have calculated structures and binding energies of a $\mathrm{Ga}$ adatom on Ga-face $\mathrm{GaN}$ (0001) surface and found that the Ga adatom is adsorbed on top of $\mathrm{N}$ atoms (T4 site [10]). Relying on the electron counting rule (ECR) $[18,19]$, the coverage of Ga adatoms is expected to be 0.25 where all the Ga dangling bond become unoccupied. Then we consider $2 \times 2$ lateral periodicity with one additional $\mathrm{Ga}$ atom at $\mathrm{T} 4$ site is a representative of the Ga-rich GaN(0001). On this Ga-rich surface, we explore stable adsorption sites for an $\mathrm{NH}_{3}$ molecule. We have found two possibilities (Fig. 1): One is on the top Ga atom (T1 site) and the other is on the Ga adtom ( $\mathrm{T}_{\mathrm{ad}}$ site hereafter) [10]. As shown in Fig. 1, the strong bond formation between the $\mathrm{N}$ atom in $\mathrm{NH}_{3}$ and the top $\mathrm{Ga}$ atom is evident. This is the reason for the stability of the two configurations. It is noteworthy that both the $\mathrm{T}_{\mathrm{ad}}$ and the $\mathrm{T} 1$ configurations satisfy the ECR with the $\mathrm{Ga}$ dangling bond unoccupied. Furthermore, we have found that the Ga-Ga bonds in the structures are rather weak, as is shown by the smaller isovalue surfaces. This weak spots may be a candidate place for the $\mathrm{N}$ incorporation (see below).

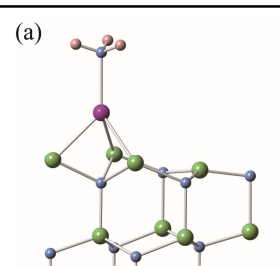

(c)

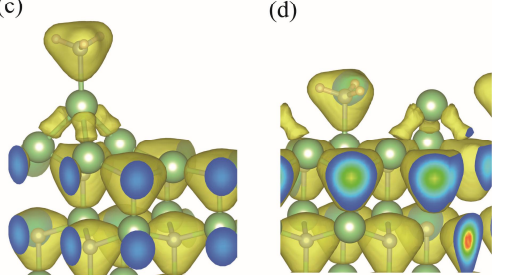

Fig. 1. Stable adsorption structure and corresponding electron density of $\mathrm{NH}_{3}$ at $\mathrm{T}_{\text {ad }}$ site [(a) and (c)] and at $\mathrm{T} 1$ site [(b) and (d)] on the Ga-rich $2 \times 2$ GaN (0001) surface. Green (large), blue (small), salmon pink (small) and burgandy (large) balls depict Ga, N, H atoms and Ga adatoms, respectively. Electron density is represented by yellow isovalue surfaces and by contour color plots at the cross-sections. (after Bui et al. [10])

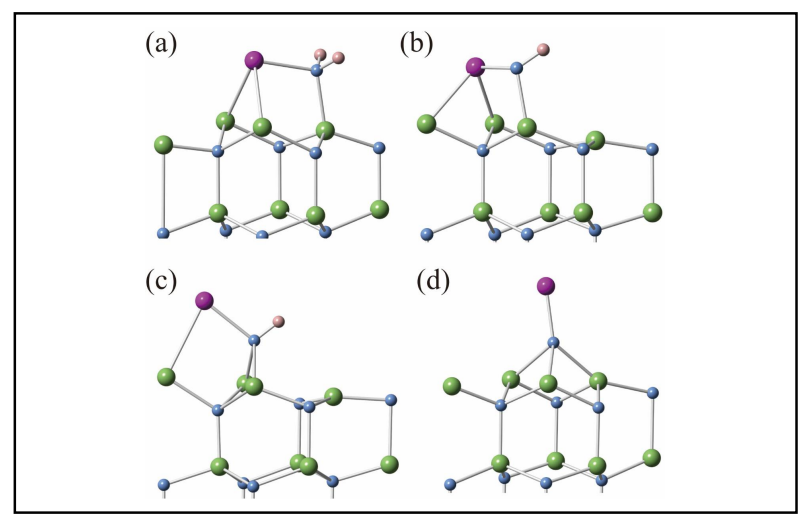

Fig. 2. Stable adsorption structures of $\mathrm{NH}_{\mathrm{x}}$ units on the Ga-rich $\mathrm{GaN}(0001)$. (a) $\mathrm{NH}_{2}$ on the $\mathrm{T} 1$ site, (b) $\mathrm{NH}$ on the $\mathrm{T} 1$ site, (c) $\mathrm{NH}$ on the BR site, and (d) substitutional adsorption of $\mathrm{N}$. The color code is the same as in Fig. 1. (after Bui et al. [10])
Behaviors of other species, $\mathrm{NH}_{2}, \mathrm{NH}$ and $\mathrm{N}$, are also important since they may be generated by the reactions of $\mathrm{NH}_{3}$ on the surface. We have thus explored the most stable sites for those species. Figure 2 shows the obtained stable structures. For $\mathrm{NH}_{2}$, we have found that the most stable site is the $\mathrm{T} 1$ site with the binding energy being $3.96 \mathrm{eV}$ [Fig. 2(a)]. Surprisingly, the $\mathrm{NH}_{2}$ unit intervenes in the Ga-Ga bond after the geometry optimization for the $\mathrm{NH}_{2}$ at $\mathrm{T} 1$ site: i.e., almost barrierless destruction of the weak Ga-Ga bond. We have also found that the $\mathrm{NH}$ unit is adsorbed at several sites including $\mathrm{T} 1$ and the bridge sites (BR site) and then intervenes in the Ga-Ga bond again with an undetected energy barrier [Fig. 2 (b) and (c)]. Another surprise for the $\mathrm{N}$ atom is its substitutional adsorption. The $\mathrm{N}$ atom approaches the $\mathrm{Ga}$ adatom, then destruct the bonds between the Ga adatom and the top Ga atoms spontaneously, and forms a Ga-N-Ga3configuration [Fig. 2 (d)]. This again indicates the weakness of the Ga-Ga bond.

The calculations for $\mathrm{NH}_{2}$ and $\mathrm{NH}$ reported above show that the weak Ga-Ga bond is likely to be the reaction site. Hence we next explore a pathway and its corresponding energy barrier for the reaction,

$$
\left[\mathrm{NH}_{3} \text { on } \mathrm{T}_{\mathrm{ad}}\right] \rightarrow[\mathrm{NH} \text { at } \mathrm{T} 1]+\mathrm{H}_{2}
$$

using the HPC method. Figure 3 shows the obtained energy profile of the reaction from $\mathrm{NH}_{3}$ at the Tad [Fig. 1 (a)] toward $\mathrm{NH}$ near the T1 [Fig. 2(b)] with the $\mathrm{H}_{2}$ molecule being desorbed. It is noteworthy that the final configuration satisfies ECR with the $\mathrm{N}$ dangling bond being occupied by 2 electrons and the two Ga dangling bonds being unoccupied. The energy barrier for this reaction of this $\mathrm{NH}_{3}$ decomposition and the subsequent $\mathrm{NH}$ incorporation in the $\mathrm{GaN}$ bond network is calculated to be $0.63 \mathrm{eV}$. This value is surprisingly small in spite that the reaction involves the cleavage of a Ga-Ga bond. The distance from the incorporated $\mathrm{N}$ of the $\mathrm{NH}$ unit to the two Ga atoms are $1.90 \AA$ and $1.92 \AA$, indicating that new Ga$\mathrm{N}$ bonds are already formed.

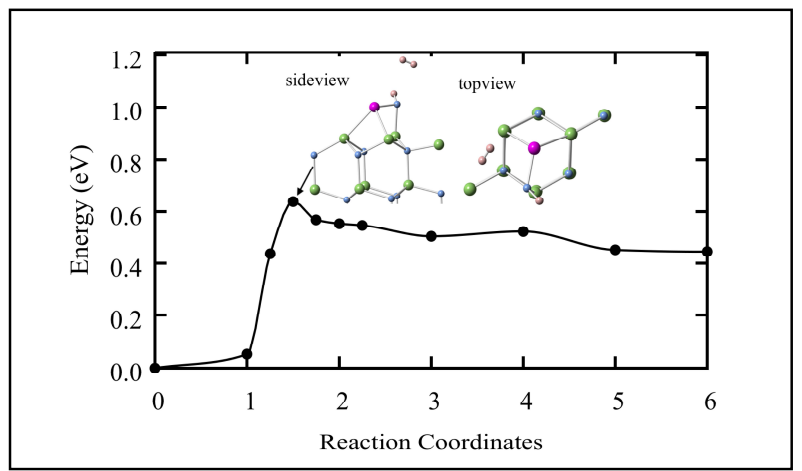

Fig. 3. Calculated energy profile for the reaction of the $\mathrm{NH}_{3}$ decomposition and the NH incorporation (see text) on the Ga-rich $\mathrm{GaN}(0001)$ growing surface. The transition-state geometry is also shown. .(after Bui et al. [10])

The next issue is how the hydrogen atoms are desorbed in a form of $\mathrm{H}_{2}$ and leaves the $\mathrm{N}$ atom surrounded by four neighboring Ga atoms. We have explored several possibilities [10] and found a plausible reaction in which the -(Ga adatom)$\left(\mathrm{NH}_{2}\right)$-Ga- structure [Fig. 2 (a)] becomes the incorporated $\mathrm{N}$ being four-fold coordinated with Ga atoms [Fig. 2 (d)]. We have identified the transition state geometry and the calculated energy barrier is $2.0 \mathrm{eV}$. Examining the energy profile, we have found that this is the energy cost to make the final state 
with $\mathrm{H}_{2}$ being desorbed. Hence, when the energy of the final state is lowered, the reaction is likely to occur. This indeed occurs in the typical growth conditions: the hydrogen chemical potential at $1300 \mathrm{~K}$ in the gas phase is evaluated to be $-2.1 \mathrm{eV}$, considering translation, vibration and rotation motions of $\mathrm{H}_{2}$. This value of the chemical potential compensates for the zero-temperature energy barrier computed above. Then the whole reaction of the formation of $\mathrm{GaN}$ unit and the $\mathrm{H}_{2}$ desorption that we have found is possible in GaN MOVPE.

\section{Ga-Ga Weak Bonds at Surface Steps of GAN(0001)}

The epitaxial growth is usually conducted on $\mathrm{GaN}(0001)$ surface inclined by less than a few degrees toward either [1120] or [1-100] direction. Such vicinal surfaces consist of terraces and steps and the reactions of the decomposition and the incorporation discussed above occur at the step edges especially in the step-flow growth. We have examined all the possible mono-bilayer atomic steps by the RSDFT calculations and obtained the stable edge structures and the formation energies. Fig. 4 shows the most stable atomic steps toward [11-20] direction. The most prominent feature is the existence of weak bonds between upper- and the lower-terrace $\mathrm{Ga}$ atoms. Ga adatom also participate in the formation of the weak bonds. These weak Ga-Ga bonds are expected to be stages of the epitaxial growth.

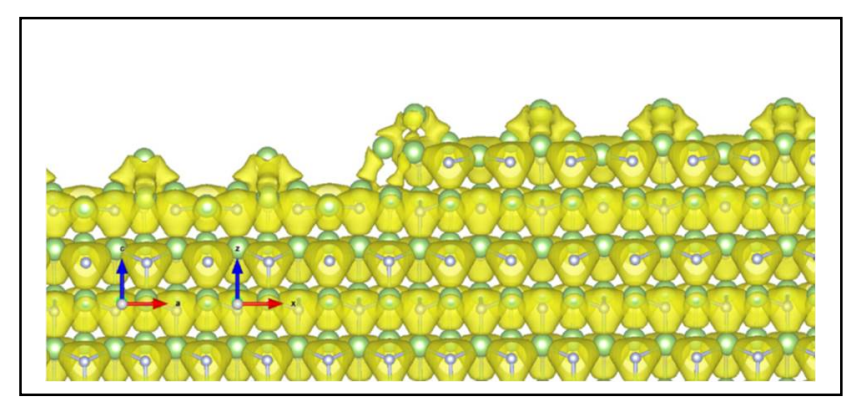

Fig. 4. Side view of a stable step structure of the [11-20]-inclined GaN (0001) vicinal surface under the Ga-rich condition. Green(large) and violet (small) balls depict $\mathrm{Ga}$ and $\mathrm{N}$ atoms, respectively. Electron density is repreented by the yellow isovalue surface.

\section{Surface Ga AdATOMS: TWO-DimenSIONAL LIQUIDS}

The growth temperature of GaN MOVPE is about $1300 \mathrm{~K}$. The behavior of surface atoms at atomistic level is totally unknown. To respond to this challenge, we have performed CPMD simulations for Ga-rich GaN(0001) surface. To allow the migration of $\mathrm{Ga}$ adatoms with long distance, we use $4 \times 4$ lateral periodicity for our simulation cell. (The $4 \times 4$ is obviously insufficient to perform more realistic simulations which are now in progress.)

A stable structure was first obtained via standard geometry optimization. Then this system was gradually heated up from room temperature to $1300 \mathrm{~K}$. During this stage, we monitored the behavior of the system at each temperature plateau by computing the pair correlation functions (PCFs):

$$
g(r)=\frac{V}{4 \pi r^{2} N^{2}}\left\langle\sum_{i} \sum_{j \neq i} \delta\left(r-r_{i j}\right)\right\rangle,
$$

being $N$ the number of atoms contained in a volume $V$ and $r_{i j}$ the interatomic distance. The average is computed on the trajectory produced by the dynamical simulation and, since both positions and velocities become simultaneously available, another quantity describing the dynamics, the diffusion coefficient can be directly obtained upon integration of the velocity-velocity autocorrelation function [20,21]:

$$
D=\frac{1}{3} \int_{0}^{t \rightarrow \infty}\left\langle\frac{1}{N} \sum_{I=1}^{N} v_{I}(t) v_{I}(0)\right\rangle d t
$$

At room temperature, as shown in the bottom panel of Fig. 5, a sharp peak at $6.4 \AA$ in the PCF is the signature of the relative high stability of $\mathrm{Ga}$ adatoms $\left(\mathrm{Ga}_{\mathrm{ad}}\right)$ on the sites where they have been placed. However, at increasing temperature, this peak broadens significantly from 500 to $1100 \mathrm{~K}$. At $1300 \mathrm{~K}$, the $\mathrm{Ga}_{\mathrm{ad}}$ undergoes the large fluctuation and the disruption of the weak bond between the $\mathrm{Ga}_{\mathrm{ad}}$ and the top surface $\mathrm{Ga}$ on the (0001) takes place. Then, $\mathrm{Ga}_{\mathrm{ad}}$ is dislodged and starts migrating on the surface. This result is well represented by the $\mathrm{Ga}_{\mathrm{ad}}-\mathrm{Ga}_{\mathrm{ad}} \mathrm{PCF}$ at $1300 \mathrm{~K}$ in the range from from 2.0 to $6.5 \AA$ with a peak centered at 2.6-2.7 $\AA$. This peak is a clear evidence of the formation of chemical bonds between two $\mathrm{Ga}_{\mathrm{ad}}$ atoms and its corresponding spread accounts rather well for all the possible $\mathrm{Ga}_{\mathrm{ad}}-\mathrm{Ga}_{\mathrm{ad}}$ chemical bonds, from single $(\sim 2.59 \AA)$ to double $(\sim 2.41-2.25 \AA)$ and triple $(\sim 2.45-2.21 \AA)$ bonds. The long tail extending rather uniformly up to the borders of the simulation cell, visible in the upper panel of Fig. 5 resemble indeed what can be observed in non-polar liquids [22].

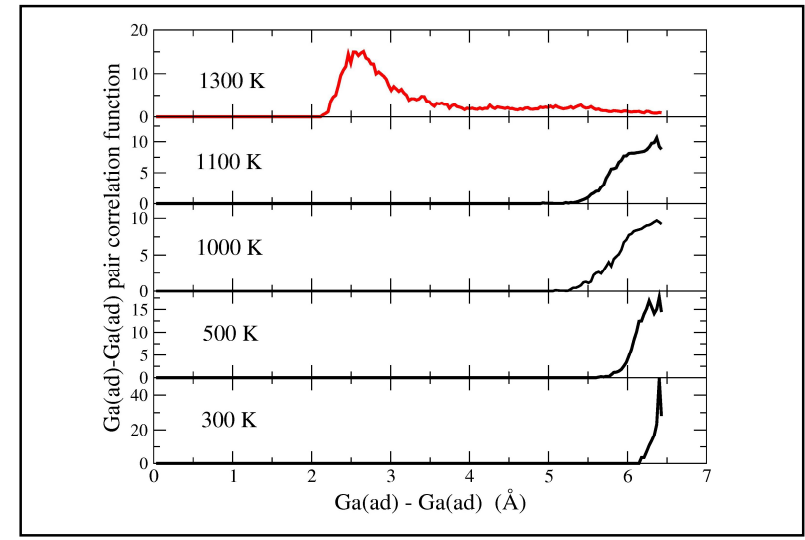

Fig. 5. Evolution of the $\mathrm{Ga}(\mathrm{ad})-\mathrm{Ga}(\mathrm{ad})$ pair correlation function (equation (2)) during the heating process. The initial distance of $6.4 \AA$ corresponds to the relaxed system from which dynamical simulations were started. (after Bui et al. [12])

What can be inferred from the analysis of the PCFs is that $\mathrm{Ga}_{\mathrm{ad}}$ is destabilized by the high temperature, starts wandering on the $\mathrm{GaN}$ (0001) surface and, due to their relatively high density, start clustering. Such an enhanced mobility is confirmed by the diffusion coefficient $D$ computed at the various temperatures explored (Fig. 6). A feature that can be immediately remarked is that, at variance with lower temperatures, $D$ deviates from the Arrhenius behavior, when $T=1300 \mathrm{~K}$. This increased diffusivity corroborates the evidence about the enhanced mobility of $\mathrm{Ga}_{\mathrm{ad}}$, which behaves like a liquid state, thus providing further support to the picture offered by the PCFs. Indeed, if we neglect the value of the diffusivity at $1300 \mathrm{~K}$, we can accommodate all the points of Fig. 4 in the Arrhenius plot with the diffusion barrier of 0.28 $\mathrm{eV}$ and the pre-exponential factor of $7.94 \times 10^{-5} \mathrm{~cm}^{2} / \mathrm{s}$. Instead, at $1300 \mathrm{~K}$ the $\mathrm{Ga}_{\mathrm{ad}}$ is destabilized and the system shows a liquid-like two-dimensional character with a sudden increase of $D$ arising and reaching a value of $0.492 \times 10^{-4} \mathrm{~cm}^{2} / \mathrm{s}$ which amounts to one order of magnitude higher than the value of $D$ $\left(0.382 \times 10^{-5}\right)$ at $1100 \mathrm{~K}$. 


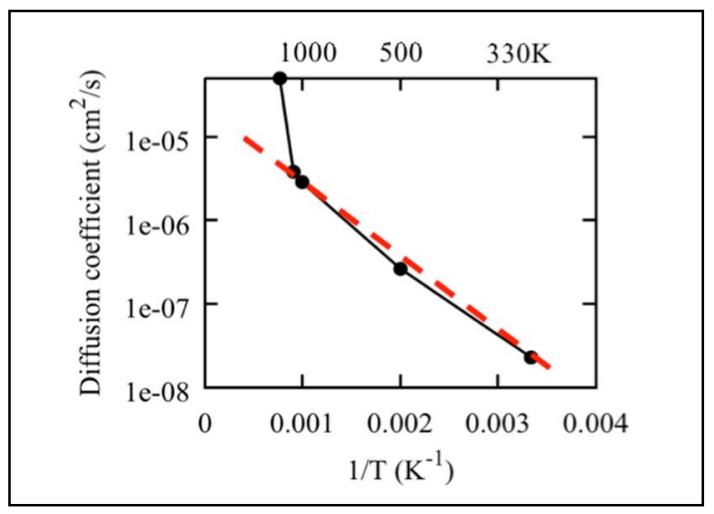

Fig. 6. Diffusion coefficient of a Ga adatom on Ga-rich GaN (0001) surface as a function of the inverse temperature $1 / \mathrm{T}$ for the temperature range $(300$ $1300 \mathrm{~K}$ ) investigated in the present work. The dashed red line shows the Arrhenius plot obtained by excluding the point at $1300 \mathrm{~K}$, where the system deviates from the standard trend. (after Bui et al. [12])

The epitaxial growth has been so far believed to occur by destruction of individual chemical bonds on the solid surface and the subsequent formation of new chemical bonds with arriving atomic and molecular species. However, the finding of the two-dimensional liquid-like phase leads to a new picture: At the growth temperature, the solid Ga-covered surface transforms to a two-dimensional liquid surface; the $\mathrm{N}$ species then easily intervenes into the subsurface region and bridges the $\mathrm{Ga}$ adatom in the liquid phase and the $\mathrm{Ga}$ atom in the sub surface layer. This is the formation of a new Ga-N network. This picture is consistent with a well-known fact that the temperature range in which the thin films grow is very narrow: The lower limit of this temperature range is the temperature at which the liquid-phase is formed, whereas the upper limit is the temperature at which the liquid evaporates into the gas phase.

\section{SUMMARY}

In summary, we have performed the DFT-based totalenergy electronic-structure calculations and CPMD simulations to clarify atomic reactions during the GaN MOVPE. We have found that the formation of the Ga-Ga weak bond in the stable structures and also in the novel liquid phase causes the $\mathrm{NH}_{3}$ decomposition and the $\mathrm{N}$ incorporation, thus being essential for the epitaxial growth.

\section{ACKNOWLEDGMENT}

The work was supported by MEXT(Japan) programs on "Post-K Supercomputer", "Supercomputer Fugaku" and "Next Generation Semiconductors for Energy-Saving Society (Contract No = JPJ005357)", and by Grants-in-aid under contract No. $18 \mathrm{H} 03873$.

\section{REFERENCES}

[1] P. Hohenberg and W. Kohn, "Inhomogeneous electron gas" Phys. Rev. 136, B864-B871 (1964).

[2] W. Kohn and L. J. Sham, "Self-consistent equations including exchange and correlation effects" Phys. Rev. 140, A1133-A1138 (1965).

[3] e.g., R. O. Jones, "Density functional theory: Its origins, rise to prominence, and future” Rev. Mod. Phys. 87, 897-923 (2015).

[4] R. Car and M. Parrinello, "Unified approach for molecular dynamics and density-functional theory" Phys. Rev. Lett. 55, 2471-2474 (1985).

[5] D. Marx and J. Hutter, "Ab Initio Molecular Dynamics (Cambridge University Press, Cambridge, 2009).

[6] M. Boero and A. Oshiyama, Encycl. Nanotechnol (Springer, Netherlands, Dordrecht, 2015), pp. 1-10.
[7] See http://www.top500.org/ for TOP 500 supercomputer site.

[8] See http://computics-material.jp/ for Materials Design through Computics.

[9] H. Amano, "Nobel Lecture: Growth of GaN on sapphire via lowtemperature deposited buffer layer and realization of p-type GaN by $\mathrm{Mg}$ doping followed by low-energy electron beam irradiation" Rev. Mod. Phys. 87, 1133-1138 (2015).

[10] K. M. Bui, J.-I. Iwata, Y. Kangawa, K. Shiraishi, Y. Shigeta, and A. Oshiyama, "A reaction pathway of surface-catalyzed ammonia decomposition and nitrogen incorporation in epitaxial growth of gallium nitride" J. Phys. Chem. C 122, 24665-24671 (2018).

[11] K. M. Bui, J.-I. Iwata, Y. Kangawa, K. Shiraishi, Y. Shigeta, and A. Oshiyama, "First-principle study of ammonia decomposition and nitrogen incorporation on the GaN surface in metal organic vapor phase epitaxy” J. Cryst. Growth 507, 421-424 (2019).

[12] K. M. Bui, M. Boero, K. Shiraishi, and A. Oshiyama, "A twodimensional liquid-like phase on Ga-rich GaN (0001) surfaces evidenced by first principles molecular dynamics" Jpn. J. Appl. Phys. 59, SGGK04 (2020).

[13] W. Saito, Y. Takada, M. Kuraguchi, K.Tsuda, I. Omura, T. Ogura, and H. Ohashi, "High breakdown voltage AlGaN-GaN Power FEMT Design and high current density switching behavior" IEEE Transactions on Electron Devices 50, 2528-2531 (2003).

[14] K. Shiraishi, K. Sekiguchi, H. Shirakawa, K. Chokawa, M. Araidai, Y. Kangawa, and K. Kakimoto, "First principles and thermodynamics studies on metal organic vapor phase epitaxy of GaN" ECS Transactions, 80, 295-301 (2017).

[15] A. Kusaba, Y. Kangawa, P. Kempisty, H. Valencia, K. Shiraishi, Y. Kumagai, K. Kakimoto, A. Koukitu, Jpn. J. Appl. Phys. 56, 070304 (2017).

[16] K. Nagamatsu, S. Nitta, Z. Ye, H. Nagao, S. Miki, Y. Honda, and H. Amano, "Decomposition of trimethylgallium and adduct formation in a metalorganic vapor phase epitaxy reactor analyzed by high-resolution gas monitoring system" 254, 1600737 (2017).

[17] L. C. Grabow, J. J. Uhlrich, T. F. Kuech, and M. Mavrikakis, "Effectiveness of in situ NH3 annealing treatments for the removal of oxygen from GaN surfaces" Surf. Sci. 603, 387-399 (2008).

[18] J. P. Perdew, K. Burke, and M. Ernzerhof, "Generalized Gradient Approximation Made Simple” Phys. Rev. Lett. 77, 3865-3868 (1996).

[19] N. Troullier and J. L. Martins, "Efficient pseudopotentials for planewave calculations” Phys. Rev. B 43, 1993-2006 (2010).

[20] S. G. Louie, S. Froyen, and M. L. Cohen, "Nonlinear ionic pseudopotentials in spin-density-functional calculations" Phys. Rev. B 26, 1738-1742 (1982).

[21] J.-I. Iwata, D. Takahashi, A. Oshiyama, T. Boku, K. Shiraishi, S. Okada, and K. Yabana, "A massively-parallel electronic-structure calculations based on real-space density functional theory" J. Comp. Phys. 229, 2339-2363 (2010).

[22] Y. Hasegawa et al., Int. J. High Performance Computing Applications 28, 335-355 (2014).

[23] CPMD, http://www.cpmd.org/, Copyright IBM Corp 1990-2019, Copyright MPI fur Festkoerperforschung Stuttgart 1997-2001.

[24] K. Shiraishi, "A new slab model approach for electronic structure calculation of polar semiconductor surface" J. Phys. Soc. Jpn. 59, 3455-3458 (1990).

[25] S. Jeong and A. Oshiyama, "Adsorption and diffusion of Si adatom on hydrogenated Si(100) surfaces” Phys. Rev. Lett. 79, 4425-4428 (1997).

[26] D. J. Chadi, "Atomic Structure of GaAs(100)-(2x1) and $(2 \times 4)$ Reconstructed Surfaces” J. Vac. Sci. Tecn. A 5, 834-837 (1987).

[27] M. D. Pashley, "Electron counting model and its application to island structures on molecular-beam epitaxy grown $\mathrm{GaAs}(001)$ and ZnSe(001)" Phys. Rev. B 40, 10481-10487 (1989).

[28] M. S. Green, "Markoff random processes and the statistical mechanics of time-dependent phenomena. II. Irreversible processes in fluids" J. Chem. Phys. 22, 398-413 (1954).

[29] R. Kubo, "Statistical-mechanical theory of irreversible processes. I. General theory and simple applications to magnetic and conduction problems"J. Phys. Soc. Jpn 12, 570-586 (1957).

[30] Y. Zhao, Z. Wu, and W. Liu, "Theoretical and analytical radial distribution function for non-polar mixtures" Physica A 390, 28122818 (2011). 
